\title{
An Awareness of Asperger's Syndrome Subcultures as an Answer to Surplus Suffering
}

\section{Benjamin Sperling}

University of Alberta

INvoke Sociology Undergraduate Journal 


\begin{abstract}
Asperger Syndrome (AS) as a diagnosis and as a community has been heavily debated in its form across both medical and AS collectives. The cornerstone of many of these discourses has been around how stakeholders and special interest groups can work together to the benefit of the AS community at large. This paper sought to uncover the proper union of perspectives to promulgate the best outcome for those identified, or self-identified, under the AS label. By exploring both the medical and AS community perspective separately, a four-part argument was conceived showcasing the creation of AS as a recognized label; the subcultural groups born from this label; how discourses insensitive to theses varying groups can catalyze 'surplus suffering'; and how subculture led discourses can bypass this surplus suffering. 'The AS Mood Disorder Synthesis Loop' was proposed as model of harm through which surplus suffering takes form.
\end{abstract}

\title{
Introduction
}

I was four when I was diagnosed with Asperger's Syndrome (AS). This label haunted me in my elementary school experience where I experienced much personal trauma from wellintending adults and not so well-intending kids. I was different. I liked to keep to myself and often engaged in solitary activities like video games and playing Lego. I had a few friends, but never felt deeply connected to them... perhaps the reason for this is that we all kept to our solitary activities, just within each others' presences. I was unable to speak properly until I was 4 and struggled to conceive the meanings behind metaphors and other figurative language. Despite all of these quirks, I was happy. This was until I started grade one when the school was informed about my apparent disorder and so the "accommodating" began.

I was forced to ride a special bus that was separate from the normal kids. I was frequently handed detentions for social infractions that other children were able to get away with, 
like being too loud. I was challenged when I did well on tests, even being accused of cheating on a few occasions since it was too unbelievable to my instructors to believe I could do well. I was even banned from competing in races on behalf of the school despite being the fastest runner in my grade. The support I was offered consisted of me having to go to special classes and meetings with children who had a whole menagerie of psychological problems, each differing in severity and symptoms from the other. My recollection was of always feeling out of place at such meetings and embarrassed that I had to be pulled from class to attend to them. This persisted until I switched schools in grade 6 whereupon I started anew with a fresh slate. Now, I enjoy a healthy menagerie of friends and success within my social abilities. The apparent weirdness of my earlier years all but a shadow in my young adult life.

The experience I just explored defined much of my early life and development into posterity. The whole experience left me feeling indignant for the longest time. Hence, I was inspired to research more into AS. I had the intention of revealing it as an unnecessary institutional weapon against diverse youth and how the institutions at play were abusive and narrow-minded. However, what I came across were perspectives that conflicted with mine, a medical community that had largely agreeable approaches, and an ignited debate over the very meaning of AS itself.

The patterns I saw in the literature led me to some fascinating conclusions around our institutional and personal relationships with AS. I have conceived a four-part argument around these patterns that I shall reveal as I move forward. The ultimate purpose of this paper is to point out how institutional harm is generated around AS and how best we can mitigate such harm through a sociological awareness of AS as a community. 
To begin, I shall explore AS and its meanings and consequences through the lens of two communities: the medical community and the AS community. Upon finishing this exploration, I shall make a four-part argument around AS as a cultural identity and what discourses around AS have been blind to. Upon revealing this blindness, I shall explore the ramifications of this lack of awareness on the AS community and how we can best address such issues to minimize harm to the community itself.

\section{The Medical Community's Perspective}

The current DSM-V observes AS under the Autism Spectrum Disorder (Spillers et al., 2014). This spectrum is characterized by two main features: 1) deficits in social interaction, communication, and comprehension across multiple contexts and 2) repetitive, restricted patterns of behaviours, interests, and activities. These conditions must be present from early on in an individual's life, placing autism spectrum disorders under pervasive developmental disorders (Spillers et al., 2014). AS is considered as high functioning autism which is the least severe disorder of the autism spectrum. There are what are known as a triad of social impairments that characterize the disorder: 1) issues in comprehension and communication, both verbal and nonverbal; 2) issues in two-way communication; 3) issues in engaging in spontaneous, nonregimented behaviours, activities, and ways of thinking (Tantam \& Girgis, 2009).

Biologically speaking, AS is strongly hereditary though its causes are still debated (Tantam \& Girgis, 2009). Its origins are believed to be multimodal and involve multiple genes with features present across them different from individuals without AS (Warrier et al., 2013; Wang et al., 2016; Runswick-Cole, 2014). No definitive link has yet been strongly supported between biology and the expression of AS in others. What is not in question is the difference in brain scans between neurotypical individuals and individuals with AS. 
First, I'll define what neurotypicality is. Neurotypicality refers to the concept of there being individuals who share roughly the same biochemical, electrical, and structural elements within their brains (Rudy, 2013). Further, neurotypicality requires the emotional, cognitive, and behavioral expressions of individuals to also be generally the same. Such expressions must also adapt in similar fashions across social contexts (Rudy, 2013). Neurotypicality is commonly used synonymously with the notion of the healthy brain. Neurotypical people are seen as healthy but AS people are observed as unhealthy. AS individuals are thus compared to people deemed as neurotypical who act as controls in brain scan research. Brain scans have shown that AS individuals have significantly different electrochemical activations in their brains than neurotypical individuals (Castelli et al., 2002; Ashwin et al., 2007; Happe et al., 1996).

These studies are positioned upon AS being interpreted as harmful to people. The medical community believes that individuals along the autism disorder spectrum have difficulties forging and sustaining close friendships; find the social world threatening; and find unpredictable changes alarming (Tantam \& Girgis, 2009). Anxiety and stress thus promulgate as individuals with AS struggle to forge desirable friendships. People with AS also have an increased chance of being diagnosed with a comorbid disorder than neurotypicals would (Tantam \& Girgis, 2009).

The medical community takes a management approach to mitigating the negative impacts of AS on people afflicted with the disorder. The pathway in place is as follows: 1) accurately diagnose AS; 2) screen for comorbidity; 3) provide resources and support around social training and general education concerning AS; 4) compliment social supports with medications (Tantam \& Girgis, 2009). Concerning four, there is a history of medical professionals prescribing medications to treat AS itself, not just the comorbid diseases and disorders that may accompany it (Tantam \& Girgis, 2009). Clinical trials exist that suggest medications are useful in treating 
AS, but such results show up only for the experiments that failed to account for the placebo effect in their trials (Tantam \& Girgis, 2009). Prescribing medications for AS patients can elicit spikes in arousal that mimic the extreme reactions observed in cerebro-vascular episodes and dementia (Carota et al., 2001). The medical community generally advises against medicating individuals with AS as a means of managing their symptoms.

\section{The Asperger's Syndrome Community's Perspective}

The perspectives of the AS community are explored through the lens of sociological research and the qualitative data it has uncovered across time. Jones \& Meldal (2001) isolated five distinct themes that individuals with AS identify as characterizing their lives: 1) awareness of communication/comprehension difficulties; 2) a desire for relationships; 3) attempts to fit in using role-play; 4) a supportive community; 5) the benefits of the internet. Themes one through three propagate a great deal of anxiety for individuals with AS, particularly over prolonged periods of time (Jones \& Meldal, 2001). Jennes-Coussens and her partners (2006) discovered that individuals with AS had significantly lower qualities of life both physically and socially with fewer positive experiences across relationships and work.

Regarding the 'supportive community' theme, sites like Autistic Self-Advocacy Network (ASAN), Autism Network International (ANI), and Wrong Planet explore AS related concepts ranging from exploring life in a neurotypical world to rebranding the very image of AS itself (Spillers et al., 2014). These various topics fulfill the needs of a vast and diverse group of people all under the AS umbrella. These online communities are not without their conflicts and controversies. Two separate debates remain at large in the community: 1) concern over AS being pooled under autism spectrum disorders in the DSM-V and 2) the neurotypicality versus neurodiversity debate. 
Concerning the first controversy, some individuals have embraced the change and call themselves "spectrumites" whereas others remain suspicious who identify as "aspies" (Giles, 2013). Aspies are concerned about: how to identify themselves; reduced support and resources; being silenced; and promoting strategies of advocacy over curative measures (Spillers et al., 2014). Some aspies are concerned about pharmaceutical manipulations behind the new DSM-V change, but the majority are critical of psychiatry in general, referring to "hard science" as the eventual means of disentangling the true definition of AS (Giles, 2013).

The neurotypicality versus neurodiversity controversy is centered upon defining what a healthy brain is. Neurodiversity is a movement towards uncoupling the stereotypes from AS and autistic individuals by embracing a diversity in behaviours, interests, and thoughts as acceptable expressions of normal people (Spillers et al., 2014). It involves resisting and challenging what the medical community defines as "illness" around individuals with AS or autism. This extends to rejecting the curative approach of well-intending entities rooted in the neurotypical model by enforcing a culture that accepts different ways of socializing and thinking as also healthy. However, there are those within both the AS and autism communities who support the "cure movement" due to the severity of their symptoms and the consequent aid such individuals seek in coping with the world (Spillers et al., 2014).

The 'benefits of the Internet' theme consolidates information, communal belonging, and activism for the AS community. Jones \& Meldal (2001) found that AS members used the internet to collect information about their conditions and ways of managing it. Davidson (2008) suggests that AS members create their own "language games" and consequently their own culture through such communication to foster a sense of belonging. Such belonging is further fostered in how members find that online communication is more considerate, responses can be edited, and the 
difficulties of implicit communication are largely removed (Benford \& Standen, 2009).

However, institutional sites, which often function as advocacy nodes, can either position AS as a disorder that needs to be cured, e.g. The Asperger's Network, Families of Adults Afflicted with Asperger Syndrome (FAAS), or as a healthy expression of human diversity, e.g. The Global and Regional Asperger Syndrome Partnership (GRASP) (Clarke \& van Amerom, 2007). AS oriented blogs exist that seek to normalize the experiences of aspie individuals and align with the advocacy work of sites like GRASP. Not all AS oriented sites fit the needs of all individuals with AS - belonging is contingent on finding the right community (Clarke \& van Amerom, 2007).

Now that I have characterized both the medical community's and Asperger's community's perspectives, I will proceed to unpack a four-part argument that will unveil a pattern not overtly discussed in the literature.

Part 1 - The Medical Community and the Creation of the AS Social Group

The medical community is rooted in neurotypicality (Rudy, 2013). Therefore, emotional, cognitive, and behavioural expressions that exist outside of the realm of neurotypicality must be defined and minimized to reduce harm. This cultural standpoint allowed for Lorna Wing, with backing from Hans Asperger's work, to define AS based on abnormal expressions of speech patterns, knowledge banks, and social comprehension (Giles, 2013). Thus, the DSM-IV included AS in its pages for the first time in the DSM's history. This galvanized a social rippling across the Internet where websites sprung up claiming to provide advice and support for those dealing with AS (Giles, 2013). Sites designed by individuals with AS, like Wrong Planet, started to proliferate with great intensity. Individuals with AS were beginning to realize that they were not alone and now finally had a medium to unite under. 
Individuals across history have borne this condition (Giles, 2013), but only when it became identified by the medical community was the cultural experience of AS able to be reified into the disorder's name. This name was the platform upon which an entire social group came together and constructed the massive online presence observed today. The medical community, perceived people experiencing AS symptoms as atypical and developed a diagnostic name for them. In granting the AS community a name, AS members who were once isolated from one another now had a common language to connect through. This connection was mediated through the Internet and visibalized an entire group of people as distinct and unique from neurotypical society. Thus, the medical community created the AS social group.

\section{Part 2 - The Four AS Subgroups}

The AS community swiftly became saturated with a diverse population of members, each with their own experiences and interpretations of AS (Giles, 2013). Specifically, communities formed online hold competing perspectives across the two controversies explored earlier: neurotypicality versus neurodiversity and aspies versus spectrumites (Giles, 2013; Spillers et al., 2014). The neurodiversity movement has existed since the conception of AS (Giles, 2013), but the aspie versus spectrumite debate only became relevant in 2010 when details about the DSM-V were first released (Spillers et al., 2014). Various sites around AS and autism were thus established for different subgroups within the AS group (Clarke \& van Amerom, 2007).

I argue that there are four subgroups that exist under the AS social group: 1) aspie/neurotypicality (AT), 2) aspie/neurodiversity (AD), 3) spectrumite/neurotypicality (ST), and 4) spectrumite/neurodiversity (SD). Each group has its own associated beliefs, perceptions, and practices around AS. Thus, each group has their own respective and distinct needs. Their needs embody as the preferred frameworks these groups desire to hold discourses from, i.e. AT 
and $\mathrm{AD}$ people would prefer not to be assumed under the autism spectrum disorder in a discourse, but ST and SD people would. Mismatching discourse frameworks with AS subgroups can lead to something known as "surplus suffering" (Clarke \& van Amerom, 2007).

\section{Part 3 - Surplus Suffering and AS Subgroup Mismatching}

Surplus suffering is when harmful stereotypes and biases are promulgated through one's interactions with a specified group and directly cause the group further harm (Clarke \& van Amerom, 2007). This is quintessentially evinced in the medical context where patients and their lived experiences are medicalized to the point where their own understanding of normalcy and well-being are questioned (Clarke \& van Amerom, 2007). This pathologization can cause patients to feel degraded and their treatments to be ridden with undue mistakes, carelessness, and unkindness (Clarke \& Fletcher, 2004). In the AS context, this is present in how the interpretations of AS people and their lived experiences are often ignored. Instead, medicalized lenses are used by both medical communities and other agents to 'help' individuals with AS. By dismissing the voice of AS individuals and insisting that their unique experiences are symptoms of a medical problem, medical communities and well-intending parties alike destroy an AS individual's sense of normalcy and humanness (Giles, 2013). Disturbingly, much of the surplus suffering dealt to AS communities is through the institutions and charities positioned around helping them in the first place (Clarke \& Fletcher, 2004).

Charities often subordinate and oppress AS community groups (Clarke \& Fletcher, 2004). They do this through three primary mechanisms: the philanthropic, the heroic and the compensatory (Clarke \& Fletcher, 2004). The philanthropic approach is focused on "helping, identifying, and treating" individuals with AS. The heroic theme refers to framing AS individuals as significant in talent and moral character despite their 'condition'. Finally, the compensatory 
theme ties into the heroic theme in how it suggests that AS individuals make up for purported 'weaknesses' through 'strengths' specific to them. These mechanisms subordinate AS individuals in how they problematize their characteristics, homogenize their diverse communal needs, relegate them as pitiful dependents and reduce their features to a trade-off of strengths and weaknesses.

I argue this surplus suffering shares a relationship with the heightened comorbidity we observe among individuals with AS. For instance, Tantam (2000) showcases how individuals with AS have a heightened risk of developing anxiety and depression for a variety of reasons. The following reasons he lists are the potential aftermaths of diagnosis: 1) family tension or breakdown; 2) higher than usual rate of adverse life events; 3) victimization; and 4) awareness of difference (Tantam, 2009). Events 1, 2, and 4 are all a combination of both the AS individual's own symptoms and reactions they and their families/peers have to their diagnosis. Event 3 arrives almost purely from an assigned diagnosis. Through being victimized, the individual with AS is treated as if they have a problem that requires correcting. Consequent accommodation leading from victimization can influence an individual in ways that render them feeling alienated (Lengel, 2015). Arising emotional issues contribute to an increased risk of triggering comorbid emotional disorders like anxiety or depression (Tantam, 2000). Well-intending charities and peers that effectively oppress and subordinate individuals with AS compound onto their emotional duress.

I propose a feedback loop is created which sustains a self-fulfilling prophecy around the medicalization of AS and its heightened comorbidity. It is called the "AS Mood Disorder Synthesis Loop" (Figure 1). The culture of medicalization has enshrined neurotypicality as the reigning cultural lens we see AS through. Neurotypicality innately rejects neurodiversity as the 
correct model - invalidating AN and SN groups. Individuals with AS recognize this cultural lens and thus grapple with their identities relative to it. This defines which of the subgroups individuals within the AS community fall under. Those individuals with AS that reject neurotypicality for neurodiversity endure increased emotional problems as medical society, charities, institutions, and individuals, in general, continue to oppress and subordinate their lived experiences. Individuals who embrace the neurotypical model are arguably more prone to the negative influences of neurotypical culture as they are more willing to accept the stereotypes and biases that exist around a medicalized AS individual.

The emotional duress experienced in a neurotypical society eventually drive individuals with AS to meet medical professionals who issue diagnoses and treatments. Through their strain, individuals with AS are likely to present with comorbid disorders by the time a doctor is seen. The patient is not only diagnosed with AS, but also with any present comorbid mood disorders, further cementing the perspective that AS correlates highly with comorbidity. Such diagnoses may aid certain individuals, but they also reinforce the medicalization of individuals with AS.

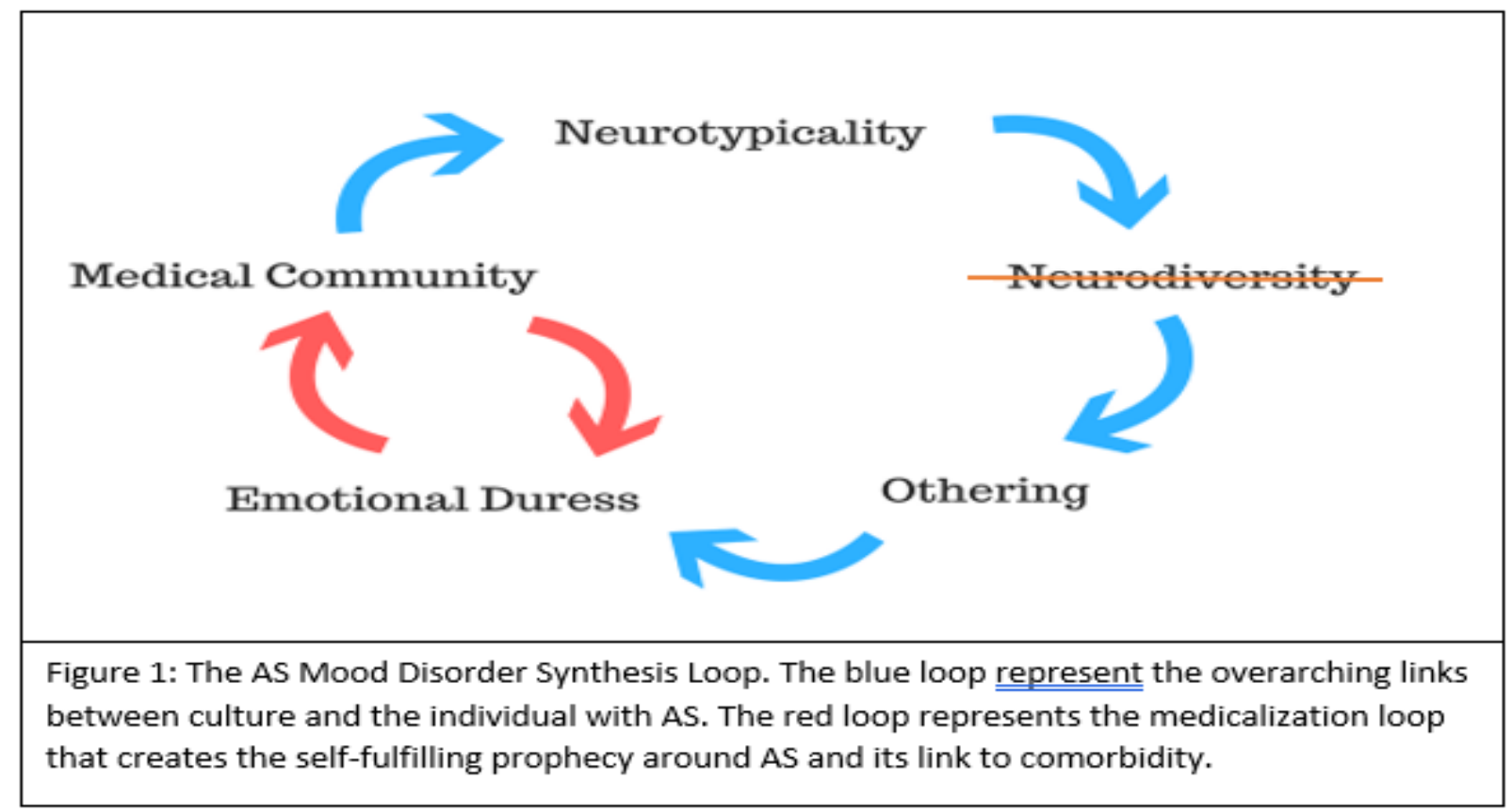


With their othering from neurotypical society enforced, feelings of isolation, frustration, and sadness promulgate, thus increasing the individual's risk of developing and enhancing mood disorders. Doctors may prescribe medications meant to treat the AS itself that can be dangerous to their patients. As explored earlier, such medications can lead to spikes in the arousal of individuals with AS and could potentially trigger the expression of comorbid disorders. This sustains the medicalization feedback loop (red) in Figure 1. Upon doctors observing the effects of this positive feedback loop, they further cement themselves in neurotypicality which strengthens it as a cultural position, thus completing the blue loop (Figure 1).

\section{Part 4 - AS Community Led and Subgroup Specific Discourses}

The AS Mood Disorder Synthesis Loop is meant to showcase two things: 1) cultural frameworks developed by those outside the AS community are harmful to all members under the AS social group and 2) AS subgroups are impacted uniquely from each other in discourses around AS. The first point addresses the first part of my final argument: discourses around individuals with AS must be lead and defined by them, not other parties. The mass promulgation of blogs written by individuals with AS speaks to a power struggle against other parties over controlling the discourse around AS people (Clarke \& van Amerom, 2007). As explored, when other institutions attempt to assume authority over defining and approaching individuals with AS, they often miss the subcultural differences found within the AS community while also subordinating them (Giles, 2013; Spillers et al., 2014; Clarke \& Fletcher, 2004). The institutions that assume authority often wield much power over how our culture manifests and so can heavily influence the collective discourse around AS (Clarke \& van Amerom, 2007).

My second point is as follows: there are multiple subgroups under the AS social group which must be recognized and accounted for when holding discourses around AS, especially in 
research. From my appraisal, the social sciences have been skilled at dissecting the cultural nuances behind AS community members and attending to multiple perspectives simultaneously. This point is mainly directed at both the medical community and the institutions or charities that are involved in discussing the AS community. The medical community almost never acknowledges the culture of neurodiversity propagating in the social science literature and continues to use language that problematizes AS. For instance, individuals with AS are referred to as "sufferers" in Tantum's (2000) paper as he assumes a clinical dissection of the issue. Ashwin and his colleagues' (2007) refer to autism as a "dysfunction of the social brain" as opposed to just saying 'differences' in the social brain. A final example that seems to express the medical community's overt biases reads as follows, "The social impairments of people with AS include deficits in empathy, self-awareness, and executive function. Many of these are quintessentially human characteristics, and the study of people with AS provides opportunities for using neuroimaging to compare people with AS and controls and identify which areas of the brain are concerned with these "higher functions."' (Tantum \& Girgis, 2009). Imagine someone with AS being told that they have "deficits" in "quintessentially human characteristics" that are regarded as "higher functions". They would likely feel degraded and dehumanized. Such individuals are being told they are deficient in essential human traits that are considered "higher" in functioning, implying they are left only with the 'lower' functioning brain regions to rely on. Overall, my four-part argument can be summarized as follows:

1) The process of medicalization designed and refined the concept of neurotypicality. Through the lens of neurotypicality, the medical community created the diagnostic name - Asperger's Syndrome. This name gave individuals who shared the cultural experiences of people with AS a name to rally under. Thus, a social group was born. 
2) The AS social group divided into four subcultures: AT, AD, ST, and SD. Each group has their own positions, beliefs, and approaches that differentiate each other, but lines can still blur.

3) Addressing any of the subcultures in a framing that does not align with their stances or homogenizing all members of the AS social group as one culture can cause surplus suffering. The "AS Mood Disorder Synthesis Loop" is a manifestation of the predominant form of surplus suffering we observe in society.

4) Surplus suffering can be avoided by keeping in mind AS community subcultures and allowing AS communities to be the 'experts' of applicable discourses. This is especially important in both research and among institutions as they are powerful platforms of advocacy and awareness around AS people.

\section{Conclusion}

Future studies should focus more on refining and uncovering the varying subgroups that exist within the AS community. The neurotypicality versus neurodiversity debate is a more potent distinction than the aspie versus spectrumite debate and so studies should prioritize accordingly. This bears the most relevance in the medical literature which should specify their groups of interest. Generally, they are speaking to AS community members who identify as spectrumites positioned upon neurotypicality. Should they wish to take a more de-medicalized approach, then directly addressing other AS community subgroups would be effective.

The medicalization loop in the "AS Mood Disorder Synthesis Loop" should also be investigated further to untangle the issue of cultural disease synthesis. The model itself can be remodified depending on changes in cultural contexts and medical conditions under 
investigation. Its core element is the concept of surplus suffering and how it sustains a disease synthesis loop.

Both the medical and social literature can profit from a more acute observance of AS community subgroups. Research holds great power and can influence entire institutions in terms of the terminologies and philosophies they use in investigating AS people at large. With this power comes a responsibility to mitigate any suffering imposed upon the AS community through precise, kind, and attentive care around the language and perspectives we utilize. 
Ashwin, C., Baron-Cohen, S., Wheelwright, S., O’Riordan, M., \& Bullmore, E. T. (2007). Differential activation of the amygdala and the 'social brain'during fearful faceprocessing in Asperger Syndrome. Neuropsychologia, 45(1), 2-14.

Bergey, B. W., \& Kaplan, A. (2010). What do social groups have to do with culture? The crucial role of shared experience. Frontiers in psychology, 1.

Benford, P., \& Standen, P. (2009). The internet: a comfortable communication medium for people with Asperger syndrome (AS) and high functioning autism (HFA)?. Journal of Assistive Technologies, 3(2), 44-53.

Carota, A., Rossetti, A. O., Karapanayiotides, T., \& Bogousslavsky, J. (2001). Catastrophic reaction in acute stroke: a reflex behavior in aphasic patients. Neurology, 57(10), 19021905.

Castelli, F., Frith, C., Happé, F., \& Frith, U. (2002). Autism, Asperger syndrome and brain mechanisms for the attribution of mental states to animated shapes. Brain, 125(8), 18391849.

Clarke, J., \& van Amerom, G. (2007). Surplus suffering': differences between organizational understandings of Asperger's syndrome and those people who claim the 'disorder. Disability \& Society, 22(7), 761-776.

Davidson, J. (2008). Autistic culture online: virtual communication and cultural expression on the spectrum. Social \& cultural geography, 9(7), 791-806.

Fox, R. C. (1977). The medicalization and demedicalization of American society. Daedalus, 922.

Fine, G. A., \& Kleinman, S. (1979). Rethinking subculture: An interactionist analysis. American journal of sociology, 85(1), 1-20.

Giles, D. C. (2014). 'DSM-V is taking away our identity': The reaction of the online community to the proposed changes in the diagnosis of Asperger's disorder. Health:, 18(2), 179-195.

Happe, F., Ehlers, S., Fletcher, P., Frith, U., Johansson, M., Gillberg, C., ... \& Frith, C. (1996). 'Theory of mind'in the brain. Evidence from a PET scan study of Asperger syndrome. Neuroreport, 8(1), 197-201.

Jennes-Coussens, M., Magill-Evans, J., \& Koning, C. (2006). The quality of life of young men with Asperger syndrome: A brief report. Autism, 10(4), 403-414. 
Jones, R. S., \& Meldal, T. O. (2001). Social relationships and Asperger's syndrome: a qualitative analysis of first-hand accounts. Journal of Learning Disabilities, 5(1), 35-41.

Lengel, L. M. (2015). A Culture of Loneliness: Asperger Syndrome Tiffany S. Campbell Bowling Green State University International Communication COMM 4090.

Mandell, D. S., Morales, K. H., Marcus, S. C., Stahmer, A. C., Doshi, J., \& Polsky, D. E. (2008). Psychotropic medication use among Medicaid-enrolled children with autism spectrum disorders. Pediatrics, 121(3), e441-e448.

Mattila, M. L., Hurtig, T., Haapsamo, H., Jussila, K., Kuusikko-Gauffin, S., Kielinen, M., ... \& Pauls, D. L. (2010). Comorbid psychiatric disorders associated with Asperger syndrome/high-functioning autism: A community-and clinic-based study. Journal of autism and developmental disorders, 40(9), 1080-1093.

Moloney, P. (2010). 'How can a chord be weird if it expresses your soul?'Some critical reflections on the diagnosis of Aspergers syndrome. Disability \& Society, 25(2), 135-148.

Rudy, L. J. (2013). What is neurotypical.

Spillers, J. L., Sensui, L. M., \& Linton, K. F. (2014). Concerns about Identity and Services among People with Autism and Asperger's Regarding DSM-5 Changes. Journal of social work in disability \& rehabilitation, 13(3), 247-260.

Tantam, D. (2000). Psychological disorder in adolescents and adults with Asperger syndrome. Autism, 4(1), 47-62.

Tantam, D., \& Girgis, S. (2009). Recognition and treatment of Asperger syndrome in the community. British Medical Bulletin, 89(1), 41-62.

Wang, Y., Picard, M., \& Gu, Z. (2016). Genetic evidence for elevated pathogenicity of mitochondrial DNA heteroplasmy in autism spectrum disorder. PLoS genetics, 12(10), e1006391.

Warrier, V., Baron-Cohen, S., \& Chakrabarti, B. (2013). Genetic variation in GABRB3 is associated with Asperger syndrome and multiple endophenotypes relevant to autism. Mol Autism, 4(1), 48.

Watabe, T., \& Suzuki, K. (2015). Internet communication of outpatients with Asperger's disorder or schizophrenia in Japan. Asia-Pacific Psychiatry, 7(1), 27-35. 